\title{
New subclasses of analytic functions
}

\author{
Basem Aref Frasin
}

Correspondence: bafrasin@yahoo. com

Faculty of Science, Department of Mathematics, Al al-Bayt University, P.O. Box 130095, Mafraq, Jordan

\section{Abstract}

For analytic functions $f(z)$ in the open unit disk $\mathcal{U}$, subclasses

$\mathcal{T}\left(\beta_{1}, \beta_{2}, \beta_{3}: \lambda\right), \mathcal{P}(\theta, \alpha)$, and $\mathcal{K}(\theta, \alpha)$ are introduced. The object of the present article is to discuss some interesting properties of functions $f(z)$ associated with classes $\mathcal{T}\left(\beta_{1}, \beta_{2}, \beta_{3}: \lambda\right), \mathcal{P}(\theta, \alpha)$, and $\mathcal{K}(\theta, \alpha)$.

Mathematics Subject Classification (2010): 30C45.

Keywords: analytic, univalent functions, Cauchy-Schwarz inequality

\section{Introduction and Definitions}

Let $\mathcal{A}$ denotes the class of the normalized functions of the form

$$
f(z)=z+\sum_{n=2}^{\infty} a_{n} z^{n}
$$

which are analytic in the open unit disk $\mathcal{U}=\{z \in \mathbb{C}:|z|<1\}$. Also, a function $f(z)$ belonging to $\mathcal{A}$ is said to be convex of order $\alpha$ if it satisfies

$$
\operatorname{Re}\left\{1+\frac{z f^{\prime \prime}(z)}{f^{\prime}(z)}\right\}>\alpha \quad(z \in \mathcal{U})
$$

for some $\alpha(0 \leq \alpha<1)$. We denote by $\mathcal{K}(\alpha)$ the subclass of $\mathcal{A}$ consisting of functions which are convex of order $\alpha$ in $\mathcal{U}$ (see, [1,2]). Further, a function $f(z)$ belonging to $\mathcal{A}$ is said to be in the class $\mathcal{P}(\alpha)$ iff

$$
\operatorname{Re}\left(z f^{\prime \prime}(z)+f^{\prime}(z)\right)>\alpha, \quad(z \in \mathcal{U}) .
$$

for some $\alpha(0 \leq \alpha<1)$.

For analytic functions $f(z)$, Uyanik and Owa [3], obtained some interesting properties for analytic functions in the subclass $\mathcal{A}\left(\beta_{1}, \beta_{2}, \beta_{3} ; \lambda\right)$ defined by

$$
\begin{gathered}
\left|\beta_{1} z\left(\frac{f(z)}{z}\right)^{\prime}+\beta_{2} z\left(\frac{f(z)}{z}\right)^{\prime \prime}+\beta_{3} z\left(\frac{f(z)}{z}\right)^{\prime \prime \prime}\right| \leq \lambda \\
\left(\beta_{1}, \beta_{2}, \beta_{3} \in \mathbb{C} ; \lambda>0 ; z \in \mathcal{U}\right),
\end{gathered}
$$

associated with close-to-convex functions and starlike functions of order $\alpha$. In this article, we define the following subclass of analytic functions.

Definition 1.1. A function $f(z)$ belonging to $\mathcal{A}$ is said to be in the class $\mathcal{T}\left(\beta_{1}, \beta_{2}, \beta_{3} ; \lambda\right)$, if it satisfies

(C) 2012 Frasin; licensee Springer. This is an Open Access article distributed under the terms of the Creative Commons Attribution License (http://creativecommons.org/licenses/by/2.0), which permits unrestricted use, distribution, and reproduction in any medium, provided the original work is properly cited. 


$$
\left|\beta_{1} z f^{\prime \prime}(z)+\beta_{2} z^{2} f^{\prime \prime \prime}(z)+\beta_{3} z^{3} f^{(4)}(z)\right| \leq \lambda \quad(z \in \mathcal{U}),
$$

for some complex numbers $\beta_{1}, \beta_{2}, \beta_{3}$, and for some real $\lambda>0$.

Example 1.2. Let us consider the function $f_{\gamma}(z), \gamma \in \mathbb{R}$, given by

$$
f_{\gamma}(z)=z(1+z)^{\gamma} \text {. }
$$

Then, we observe that

$$
\begin{aligned}
& \left|\beta_{1} z f_{\gamma}{ }^{\prime \prime}(z)+\beta_{2} z^{2} f_{\gamma}{ }^{\prime \prime \prime}(z)+\beta_{3} z^{3} f_{\gamma}^{(4)}(z)\right| \\
& \quad=\left|\sum_{n=2}^{\infty} n(n-1)\left(\begin{array}{c}
\gamma \\
n-1
\end{array}\right)\left(\beta_{1}+(n-2) \beta_{2}+(n-2)(n-3) \beta_{3}\right) z^{n-1}\right|,
\end{aligned}
$$

where

$$
\left(\begin{array}{c}
\gamma \\
n-1
\end{array}\right)=\frac{\gamma(\gamma-1)(\gamma-2) \cdots(\gamma-n+2)}{(n-1) !}
$$

Therefore, if $\gamma=1$, then

$$
\left|\beta_{1} z f_{1}^{\prime \prime}(z)+\beta_{2} z^{2} f_{1}^{\prime \prime \prime}(z)+\beta_{3} z^{3} f_{1}^{(4)}(z)\right|=\left|2 \beta_{1} z\right| \leq 2\left|\beta_{1}\right| .
$$

This implies that $f_{1}(z) \in \mathcal{T}\left(\beta_{1}, \beta_{2}, \beta_{3} ; \lambda\right)$ for $\lambda \geq 2\left|\beta_{1}\right|$. If $\gamma=2$, then

$$
\left|\beta_{1} z f_{2}^{\prime \prime}(z)+\beta_{2} z^{2} f_{2}^{\prime \prime \prime}(z)+\beta_{3} z^{3} f_{2}^{(4)}(z)\right|=\left|4 \beta_{1} z+6\left(\beta_{1}+\beta_{2}\right) z^{2}\right| \leq 10\left|\beta_{1}\right|+6\left|\beta_{2}\right| .
$$

Therefore, $f_{2}(z) \in \mathcal{T}\left(\beta_{1}, \beta_{2}, \beta_{3} ; \lambda\right)$ for $\lambda \geq 10\left|\beta_{1}\right|+6\left|\beta_{2}\right|$. Further, if $\gamma=3$; then we have

$$
\begin{aligned}
& \left|\beta_{1} z f_{3}^{\prime \prime}(z)+\beta_{2} z^{2} f_{3}^{\prime \prime \prime}(z)+\beta_{3} z^{3} f_{3}^{(4)}(z)\right| \\
& \quad=\left|6 \beta_{1} z+18\left(\beta_{1}+\beta_{2}\right) z^{2}+12\left(\beta_{1}+2 \beta_{2}+2 \beta_{3}\right) z^{3}\right| \\
& \quad \leq 36\left|\beta_{1}\right|+42\left|\beta_{2}\right|+24\left|\beta_{3}\right| .
\end{aligned}
$$

Thus, $f_{3}(z) \in \mathcal{T}\left(\beta_{1}, \beta_{2}, \beta_{3} ; \lambda\right)$ for $\lambda \geq 36\left|\beta_{1}\right|+42\left|\beta_{2}\right|+24\left|\beta_{3}\right|$.

Now, let $\mathcal{A}_{\theta}$ denotes the subclass of $\mathcal{A}$ consisting of functions $f(z)$ with

$$
a_{n}=\left|a_{n}\right| e^{i((n-1) \theta+\pi)} \quad(n=2,3, \ldots) .
$$

Also, we introduce the subclasses $\mathcal{P}(\theta, \alpha)$ and $\mathcal{K}(\theta, \alpha)$ of $\mathcal{A}_{\theta}$ as follows:

$$
\mathcal{P}(\theta, \alpha)=\mathcal{A}_{\theta} \cap \mathcal{P}(\alpha) \quad \text { and } \mathcal{K}(\theta, \alpha)=\mathcal{A}_{\theta} \cap \mathcal{K}(\alpha) .
$$

\section{Properties of the class $\mathcal{T}\left(\beta_{1}, \beta_{2}, \beta_{3} ; \lambda\right)$}

We first prove

Theorem 2.1. If $f(z) \in \mathcal{A}$ satisfies

$$
\sum_{n=2}^{\infty} n(n-1)\left(\left|\beta_{1}\right|+(n-2)\left|\beta_{2}\right|+(n-2)(n-3)\left|\beta_{3}\right|\right)\left|a_{n}\right| \leq \lambda
$$


for some complex numbers $\beta_{1}, \beta_{2}, \beta_{3}$ and for some real $\lambda>0$, then $f(z) \in \mathcal{T}\left(\beta_{1}, \beta_{2}, \beta_{3} ; \lambda\right)$.

Proof. We observe that

$$
\begin{aligned}
& \left|\beta_{1} z f_{3}^{\prime \prime}(z)+\beta_{2} z^{2} f_{3}^{\prime \prime \prime}(z)+\beta_{3} z^{3} f_{3}^{(4)}(z)\right| \\
& \quad=\left|\sum_{n=2}^{\infty} n(n-1)\left(\beta_{1}+(n-2) \beta_{2}+(n-2)(n-3) \beta_{3}\right) a_{n} z^{n-1}\right| \\
& \quad \leq \sum_{n=2}^{\infty} n(n-1)\left(\left|\beta_{1}\right|+(n-2)\left|\beta_{2}\right|+(n-2)(n-3)\left|\beta_{3}\right|\right)\left|a_{n}\right||z|^{n-1} \\
& \quad<\sum_{n=2}^{\infty} n(n-1)\left(\left|\beta_{1}\right|+(n-2)\left|\beta_{2}\right|+(n-2)(n-3)\left|\beta_{3}\right|\right)\left|a_{n}\right| .
\end{aligned}
$$

Therefore, if $f(z)$ satisfies the inequality (2.1), then $f(z) \in \mathcal{T}\left(\beta_{1}, \beta_{2}, \beta_{3} ; \lambda\right)$.

Next, we prove

Theorem 2.2. if $f(z) \in \mathcal{T}\left(\beta_{1}, \beta_{2}, \beta_{3} ; \lambda\right)$ with $\arg \beta_{1}=\arg \beta_{2}=\arg \beta_{3}=\varphi$ and $a_{n}=1$ $a_{n} \mid e^{i((n-1) \theta-\varphi)}(n=2,3, \ldots)$, then we have

$$
\sum_{n=2}^{\infty} n(n-1)\left(\left|\beta_{1}\right|+(n-2)\left|\beta_{2}\right|+(n-2)(n-3)\left|\beta_{3}\right|\right)\left|a_{n}\right| \leq \lambda .
$$

Proof. For $f(z) \in \mathcal{T}\left(\beta_{1}, \beta_{2}, \beta_{3} ; \lambda\right)$, we see that

$$
\begin{aligned}
& \left|\beta_{1} z f_{3}^{\prime \prime}(z)+\beta_{2} z^{2} f_{3}^{\prime \prime \prime}(z)+\beta_{3} z^{3} f_{3}^{(4)}(z)\right| \\
& \quad=\left|\sum_{n=2}^{\infty} n(n-1)\left(\beta_{1}+(n-2) \beta_{2}+(n-2)(n-3) \beta_{3}\right) a_{n} z^{n-1}\right| \\
& \quad=\left|\sum_{n=2}^{\infty} n(n-1)\left(\left|\beta_{1}\right|+(n-2)\left|\beta_{2}\right|+(n-2)(n-3)\left|\beta_{3}\right|\right)\right| a_{n}\left|e^{i(n-1) \theta} z^{n-1}\right| \\
& \quad \leq \lambda .
\end{aligned}
$$

for all $z \in \mathcal{U}$. Let us consider a point $z \in \mathcal{U}$ such that $z=|z| e^{-i \theta}$.

Then we have

$$
\left.\left|\sum_{n=2}^{\infty} n(n-1)\left(\left|\beta_{1}\right|+(n-2)\left|\beta_{2}\right|+(n-2)(n-3)\left|\beta_{3}\right|\right)\right| a_{n}|| z\right|^{n-1} \mid \leq \lambda .
$$

Letting $|z| \rightarrow 1^{-}$, we obtain

$$
\sum_{n=2}^{\infty} n(n-1)\left(\left|\beta_{1}\right|+(n-2)\left|\beta_{2}\right|+(n-2)(n-3)\left|\beta_{3}\right|\right)\left|a_{n}\right| \leq \lambda .
$$

Corollary 2.3. If $f(z) \in \mathcal{T}\left(\beta_{1}, \beta_{2}, \beta_{3} ; \lambda\right)$ with $\arg \beta_{1}=\arg \beta_{2}=\arg \beta_{3}=\varphi$ and $a_{n}=1$ $a_{n} \mid e^{i((n-1) \theta-\varphi)}(n=2,3, \ldots)$, then we have

$$
\left|a_{n}\right| \leq \frac{\lambda}{n(n-1)\left(\left|\beta_{1}\right|+(n-2)\left|\beta_{2}\right|+(n-2)(n-3)\left|\beta_{3}\right|\right)} \quad(n=2,3, \ldots) .
$$


Example 2.4. Let us consider the function $f(z) \in \mathcal{T}\left(\beta_{1}, \beta_{2}, \beta_{3} ; \lambda\right)$ with $\arg \beta_{1}=\arg \beta_{2}$ $=\arg \beta_{3}=\varphi$ and

$$
a_{n}=\frac{\lambda e^{i((n-1) \theta-\phi)}}{n^{2}(n-1)^{2}\left(\left|\beta_{1}\right|+(n-2)\left|\beta_{2}\right|+(n-2)(n-3)\left|\beta_{3}\right|\right)} \quad(n=2,3 \ldots) .
$$

Then, we see that

$$
\begin{aligned}
& \sum_{n=2}^{\infty} n(n-1)\left(\left|\beta_{1}\right|+(n-2)\left|\beta_{2}\right|+(n-2)(n-3)\left|\beta_{3}\right|\right)\left|a_{n}\right| \\
= & \lambda \sum_{n=2}^{\infty} \frac{1}{n(n-1)}=\lambda \sum_{n=2}^{\infty}\left(\frac{1}{n-1}-\frac{1}{n}\right)=\lambda .
\end{aligned}
$$

Corollary 2.5. If $f(z) \in \mathcal{T}\left(\beta_{1}, \beta_{2}, \beta_{3} ; \lambda\right)$ with $\arg \beta_{1}=\arg \beta_{2}=\arg \beta_{3}=\varphi$ and $a_{n}=\mid$ $a_{n} \mid e^{i((n-1) \theta-\varphi)}(\mathrm{n}=2,3, \ldots)$, then we have

$$
|z|-\sum_{n=2}^{j}\left|a_{n}\right||z|^{n}-A_{j}|z|^{j+1} \leq|f(z)| \leq|z|+\sum_{n=2}^{j}\left|a_{n}\right||z|^{n}+A_{j}|z|^{j+1}
$$

with

$$
A_{j}=\frac{\left(\lambda-\sum_{n=2}^{j} n(n-1)\left(\left|\beta_{1}\right|+(n-2)\left|\beta_{2}\right|+(n-2)(n-3)\left|\beta_{3}\right|\right)\left|a_{n}\right|\right)}{\left.j(j+1)\left(\left|\beta_{1}\right|\right)+(j-1)\left|\beta_{2}\right|+(j-1)(j-2)\left|\beta_{3}\right|\right)}
$$

and

$$
1-\sum_{n=2}^{j}\left|a_{n}\right||z|^{n-1}-B_{j}|z|^{j} \leq\left|f^{\prime}(z)\right| \leq 1+\sum_{n=2}^{j}\left|a_{n}\right||z|^{n-1}+B_{j}|z|^{j}
$$

with

$$
B_{j}=\frac{\left(\lambda-\sum_{n=2}^{j} n(n-1)\left(\left|\beta_{1}\right|+(n-2)\left|\beta_{2}\right|+(n-2)(n-3)\left|\beta_{3}\right|\right)\left|a_{n}\right|\right)}{j\left(\left|\beta_{1}\right|+(j-1)\left|\beta_{2}\right|+(j-1)(j-2)\left|\beta_{3}\right|\right)}
$$

Proof. In view of Theorem 2.1, we know that

$$
\begin{aligned}
& \sum_{n=j+1}^{\infty} n(n-1)\left(\left|\beta_{1}\right|+(n-2)\left|\beta_{2}\right|+(n-2)(n-3)\left|\beta_{3}\right|\right)\left|a_{n}\right| \\
& \quad \leq \lambda-\sum_{n=2}^{j} n(n-1)\left(\left|\beta_{1}\right|+(n-2)\left|\beta_{2}\right|+(n-2)(n-3)\left|\beta_{3}\right|\right)\left|a_{n}\right| .
\end{aligned}
$$

Further, we note that

$$
\begin{aligned}
& j(j+1)\left(\left|\beta_{1}\right|+(j-1)\left|\beta_{2}\right|+(j-1)(j-2)\left|\beta_{3}\right|\right) \sum_{n=j+1}^{\infty}\left|a_{n}\right| \\
& \leq \sum_{n=j+1}^{\infty} n(n-1)\left(\left|\beta_{1}\right|+(n-2)\left|\beta_{2}\right|+(n-2)(n-3)\left|\beta_{3}\right|\right)\left|a_{n}\right|,
\end{aligned}
$$


which is equivalent to

$$
\begin{aligned}
\sum_{n=j+1}^{\infty}\left|a_{n}\right| & \leq \frac{\left(\lambda-\sum_{n=2}^{j} n(n-1)\left(\left|\beta_{1}\right|+(n-2)\left|\beta_{2}\right|+(n-2)(n-3)\left|\beta_{3}\right|\right)\left|a_{n}\right|\right)}{j(j+1)\left(\left|\beta_{1}\right|+(j-1)\left|\beta_{2}\right|+(j-1)(j-2)\left|\beta_{3}\right|\right)} \\
& =A_{j} .
\end{aligned}
$$

Thus, we have

$$
|f(z)| \leq|z|+\sum_{n=2}^{j}\left|a_{n}\right||z|^{n}+\sum_{n=j+1}^{\infty}\left|a_{n}\right||z|^{n} \leq|z|+\sum_{n=2}^{j}\left|a_{n}\right||z|^{n}+A_{j}|z|^{j+1}
$$

and

$$
|f(z)| \geq|z|-\sum_{n=2}^{j}\left|a_{n}\right||z|^{n}-\sum_{n=j+1}^{\infty}\left|a_{n}\right||z|^{n} \geq|z|-\sum_{n=2}^{j}\left|a_{n}\right||z|^{n}-A_{j}|z|^{j+1} .
$$

Next, we observe that

$$
\begin{aligned}
& j\left(\left|\beta_{1}\right|+(j-1)\left|\beta_{2}\right|+(j-1)(j-2)\left|\beta_{3}\right|\right) \sum_{n=j+1}^{\infty} n\left|a_{n}\right| \\
& \leq \sum_{n=j+1}^{\infty} n(n-1)\left(\left|\beta_{1}\right|+(n-2)\left|\beta_{2}\right|+(n-2)(n-3)\left|\beta_{3}\right|\right)\left|a_{n}\right| \\
& \leq \lambda \sum_{n=2}^{j} n(n-1)\left(\left|\beta_{1}\right|+(n-1)\left|\beta_{2}\right|+(n-2)(n-3)\left|\beta_{3}\right|\right)\left|a_{n}\right|,
\end{aligned}
$$

which implies that

$$
\begin{aligned}
\sum_{n=j+1}^{\infty} n\left|a_{n}\right| & \leq \frac{\left(\lambda-\sum_{n=2}^{j} n(n-1)\left(\left|\beta_{1}\right|+(n-2)\left|\beta_{2}\right|+(n-2)(n-3)\left|\beta_{3}\right|\right)\left|a_{n}\right|\right)}{j\left(\left|\beta_{1}\right|+(j-1)\left|\beta_{2}\right|+(j-1)(j-2)\left|\beta_{3}\right|\right)} \\
& =B_{j} .
\end{aligned}
$$

Therefore, we obtain that

$$
\left|f^{\prime}(z)\right| \leq 1+\sum_{n=2}^{j} n\left|a_{n}\right||z|^{n-1}+\sum_{n=j+1}^{\infty} n\left|a_{n}\right||z|^{n-1} \leq 1+\sum_{n=2}^{j}\left|a_{n}\right||z|^{n-1}+B_{j}|z|^{j}
$$

and

$$
\left|f^{\prime}(z)\right| \geq 1-\sum_{n=2}^{j} n\left|a_{n}\right||z|^{n-1}-\sum_{n=j+1}^{\infty} n\left|a_{n}\right||z|^{n-1} \geq 1-\sum_{n=2}^{j}\left|a_{n}\right||z|^{n-1}-B_{j}|z|^{j} .
$$

3. Radius problem for the class $\mathcal{P}(\theta, \alpha)$

To obtain the radius problem for the class $\mathcal{P}(\theta, \alpha)$, we need the following lemma. 
Lemma 3.1. If $f(z) \in \mathcal{P}(\theta, \alpha)$, then

$$
\sum_{n=2}^{\infty} n^{2}\left|a_{n}\right| \leq 1-\alpha .
$$

Proof. Let $f(z) \in \mathcal{P}(\theta, \alpha)$. Then, we have

$$
\begin{aligned}
\operatorname{Re}\left\{\left(z f^{\prime \prime}(z)+f^{\prime}(z)\right)\right\} & =\operatorname{Re}\left\{1+\sum_{n=2}^{\infty} n^{2} a_{n} z^{n-1}\right\} \\
& =\operatorname{Re}\left\{1+\sum_{n=2}^{\infty} n^{2}\left|a_{n}\right| e^{i((n-1) \theta+\pi)} z^{n-1}\right\} \\
& =\operatorname{Re}\left\{1-\sum_{n=2}^{\infty} n^{2}\left|a_{n}\right| e^{i((n-1) \theta)} z^{n-1}\right\}>\alpha
\end{aligned}
$$

for all $z \in \mathcal{U}$. Let us consider a point $z \in \mathcal{U}$ such that $z=|z| e^{-i \theta}$.

Then we have

$$
1-\sum_{n=2}^{\infty} n^{2}\left|a_{n}\right||z|^{n-1}>\alpha
$$

Letting $|z| \rightarrow 1^{-}$, we obtain the inequality (3.1).

Corollary 3.2. If $f(z) \in \mathcal{P}(\theta, \alpha)$, then

$$
\left|a_{n}\right| \leq \frac{1-\alpha}{n^{2}} \quad(n=2,3, \ldots) .
$$

Remark 3.3. By Lemma 3.1, we observe that if $f(z) \in \mathcal{P}(\theta, \alpha)$, then

$$
\sum_{n=2}^{\infty} n(n-1)\left|a_{n}\right| \leq \sum_{n=2}^{\infty} n^{2}\left|a_{n}\right| \leq 1-\alpha .
$$

Applying Theorem 2.1 and Lemma 3.1, we derive

Theorem 3.4. Let $f(z) \in \mathcal{P}(\theta, \alpha)$, and $\delta \in \mathbb{C}(0<|\delta|<1)$. Then the function $\frac{1}{\delta} f(\delta z) \in \mathcal{T}\left(\beta_{1}, \beta_{2}, \beta_{3} ; \lambda\right)$ for $\left(0<|\delta| \leq\left|\delta_{0}(\lambda)\right|\right.$, where $\left|\delta_{0}(\lambda)\right|$ is the smallest positive root of the equation

$$
\begin{aligned}
& \left|\beta_{1}\right| \frac{|\delta| \sqrt{2(1-\alpha)}}{\left(1-|\delta|^{2}\right)^{3 / 2}}+\left|\beta_{2}\right| \frac{|\delta|^{2} \sqrt{\left(6+18|\delta|^{2}\right)\left(1-\alpha-2\left|a_{2}\right|^{2}\right)}}{\left(1-|\delta|^{2}\right)^{5 / 2}} \\
& +\left|\beta_{3}\right| \frac{4 \sqrt{3}|\delta|^{3} \sqrt{\left.\left(1+8|\delta|^{2}+6|\delta|^{4}\right)(1-\alpha-2)\left|a_{2}\right|^{2}-6\left|a_{3}\right|^{2}\right)}}{\left(1-|\delta|^{2}\right)^{7 / 2}}=\lambda
\end{aligned}
$$

in $0<|\delta|<1$.

Proof. For $f(z) \in \mathcal{P}(\theta, \alpha)$, we see that

$$
\frac{1}{\delta} f(\delta z)=z+\sum_{n=2}^{\infty} \delta^{n-1} a_{n} z^{n}
$$


and

$$
\sum_{n=2}^{\infty} n(n-1)\left|a_{n}\right|^{2} \leq 1-\alpha
$$

Thus, to show that $\frac{1}{\delta} f(\delta z) \in \mathcal{T}\left(\beta_{1}, \beta_{2}, \beta_{3} ; \lambda\right)$, from Theorem 2.1 , it is sufficient to prove that

$$
\sum_{n=2}^{\infty} n(n-1)\left(\left|\beta_{1}\right|+(n-2)\left|\beta_{2}\right|+(n-2)(n-3)\left|\beta_{3}\right|\right)|\delta|^{n-1}\left|a_{n}\right| \leq \lambda .
$$

Applying Cauchy-Schwarz inequality, we note that

$$
\begin{aligned}
& \sum_{n=2}^{\infty} n(n-1)\left(\left|\beta_{1}\right|+(n-2)\left|\beta_{2}\right|+(n-2)(n-3)\left|\beta_{3}\right|\right)|\delta|^{n-1}\left|a_{n}\right| \\
& \leq \frac{\left|\beta_{1}\right|}{|\delta|}\left(\sum_{n=2}^{\infty} n(n-1)|\delta|^{2 n}\right)^{\frac{1}{2}}\left(\sum_{n=2}^{\infty} n(n-1)\left|a_{n}\right|^{2}\right)^{\frac{1}{2}} \\
& +\frac{\left|\beta_{2}\right|}{|\delta|}\left(\sum_{n=3}^{\infty} n(n-1)(n-2)^{2}|\delta|^{2 n}\right)^{\frac{1}{2}}\left(\sum_{n=3}^{\infty} n(n-1)\left|a_{n}\right|^{2}\right)^{\frac{1}{2}} \\
& \quad+\frac{\left|\beta_{3}\right|}{|\delta|}\left(\sum_{n=4}^{\infty} n(n-1)(n-2)^{2}(n-3)^{2}|\delta|^{2 n}\right)^{\frac{1}{2}}\left(\sum_{n=4}^{\infty} n(n-1)\left|a_{n}\right|^{2}\right)^{\frac{1}{2}} \\
& \leq \frac{\left|\beta_{1}\right|}{|\delta|}\left(\sum_{n=2}^{\infty} n(n-1)|\delta|^{2 n}\right)^{\frac{1}{2}} \sqrt{1-\alpha} \\
& +\frac{\left|\beta_{2}\right|}{|\delta|}\left(\sum_{n=3}^{\infty} n(n-1)(n-2)^{2}|\delta|^{2 n}\right)^{\frac{1}{2}} \sqrt{1-\alpha-2\left|a_{2}\right|^{2}} \\
& \quad
\end{aligned}
$$

We note that

$$
\sum_{n=0}^{\infty} x^{n}=\frac{1}{1-x}, \quad(|x|<1)
$$

thus, we have

$$
\sum_{n=2}^{\infty} n(n-1) x^{n}=\frac{2 x^{2}}{(1-x)^{3}} .
$$

Since

$$
\sum_{n=3}^{\infty}(n-2) x^{n-1}=x^{2}\left(\sum_{n=3}^{\infty}(n-2) x^{n-3}\right)=x^{2}\left(\sum_{n=3}^{\infty} x^{n-2}\right)^{\prime}=\frac{x^{2}}{(1-x)^{2}}
$$


we see that

$$
\sum_{n=3}^{\infty}(n-1)(n-2)^{2} x^{n}=x^{3}\left(\frac{x^{2}}{(1-x)^{2}}\right)^{\prime \prime}=\frac{2 x^{3}+4 x^{4}}{(1-x)^{4}} .
$$

and thus, we obtain

$$
\sum_{n=3}^{\infty} n(n-1)(n-2)^{2} x^{n}=\frac{6 x^{3}+18 x^{4}}{(1-x)^{5}}
$$

Furthermore, we have

$$
\begin{aligned}
\sum_{n=4}^{\infty}(n-1)(n-2)^{2}(n-3)^{2} x^{n} & =x^{4}\left(\sum_{n=4}^{\infty}(n-1)(n-2)^{2}(n-3)^{2} x^{n-4}\right) \\
& =x^{4}\left(\sum_{n=4}^{\infty}(n-2)(n-3) x^{n-1}\right)^{\prime \prime \prime},
\end{aligned}
$$

but

$$
\sum_{n=4}^{\infty}(n-2)(n-3) x^{n-1}=x^{3}\left(\sum_{n=4}^{\infty}(n-2)(n-3) x^{n-4}\right)=\frac{2 x^{3}}{(1-x)^{3}}
$$

thus, we have

$$
\sum_{n=4}^{\infty}(n-1)(n-2)^{2}(n-3)^{2} x^{n}=\frac{12 x^{4}+72 x^{5}+36 x^{6}}{(1-x)^{6}}
$$

which yields

$$
\sum_{n=4}^{\infty} n(n-1)(n-2)^{2}(n-3)^{2} x^{n}=\frac{48 x^{4}\left(1+8 x+6 x^{2}\right)}{(1-x)^{7}} .
$$

Therefore, from (3.3)-(3.6) with $|\delta|^{2}=x$, we obtain

$$
\begin{array}{r}
\sum_{n=2}^{\infty} n(n-1)\left(\left|\beta_{1}\right|+(n-2)\left|\beta_{2}\right|+(n-2)(n-3)\left|\beta_{3}\right|\right)|\delta|^{n-1}\left|a_{n}\right| \\
\leq\left|\beta_{1}\right| \frac{|\delta| \sqrt{2(1-\alpha)}}{\left(1-|\delta|^{2}\right)^{3 / 2}}+\left|\beta_{2}\right| \frac{|\delta|^{2} \sqrt{\left(6+18|\delta|^{2}\right)\left(1-\alpha-2\left|a_{2}\right|^{2}\right)}}{\left(1-|\delta|^{2}\right)^{5 / 2}} \\
\left|\beta_{3}\right| \frac{4 \sqrt{3}|\delta|^{3} \sqrt{\left(1+8|\delta|^{2}+6|\delta|^{4}\right)\left(1-\alpha-2\left|a_{2}\right|^{2}-6\left|a_{3}\right|^{2}\right)}}{\left(1-|\delta|^{2}\right)^{7 / 2}}
\end{array}
$$

Now, let us consider the complex number $\delta(0<|\delta|<1)$ such that

$$
\begin{aligned}
& \left|\beta_{1}\right| \frac{|\delta| \sqrt{2(1-\alpha)}}{\left(1-|\delta|^{2}\right)^{3 / 2}}+\left|\beta_{2}\right| \frac{|\delta|^{2} \sqrt{\left(6+18|\delta|^{2}\right)\left(1-\alpha-2\left|a_{2}\right|^{2}\right)}}{\left(1-|\delta|^{2}\right)^{5 / 2}} \\
& \left|\beta_{3}\right| \frac{4 \sqrt{3}|\delta|^{3} \sqrt{\left(1+8|\delta|^{2}+6|\delta|^{4}\right)\left(1-\alpha-2\left|a_{2}\right|^{2}-6\left|a_{3}\right|^{2}\right)}}{\left(1-|\delta|^{2}\right)^{7 / 2}}=\lambda .
\end{aligned}
$$


If we define the function $h(|\delta|)$ by

$$
\begin{aligned}
h(|\delta|)= & \left|\beta_{1}\right||\delta|\left(1-|\delta|^{2}\right)^{2} \sqrt{2(1-\alpha)} \\
& +\left|\beta_{2}\right||\delta|^{2}\left(1-|\delta|^{2}\right) \sqrt{\left(6+18|\delta|^{2}\right)\left(1-\alpha-2\left|a_{2}\right|^{2}\right)} \\
& +4 \sqrt{3}\left|\beta_{3}\right||\delta|^{3} \sqrt{\left(1+8|\delta|^{2}+6|\delta|^{4}\right)\left(1-\alpha-2\left|a_{2}\right|^{2}-6\left|a_{3}\right|^{2}\right)} \\
& -\lambda\left(1-|\delta|^{2}\right)^{7 / 2},
\end{aligned}
$$

then we have $h(0)=-\lambda<0$ and $h(1)=12 \sqrt{5}\left|\beta_{3}\right| \sqrt{1-\alpha-2\left|a_{2}\right|^{2}-6\left|a_{3}\right|^{2}}>0$. This means that there exists some $\delta_{0}$ such that $h\left(\left|\delta_{0}\right|\right)=0\left(0<\left|\delta_{0}\right|<1\right)$. This completes the proof of the theorem.

\section{Radius problem for the class $\mathcal{K}(\theta, \alpha)$}

For the class $\mathcal{K}(\theta, \alpha)$, we prove the following lemma.

Lemma 4.1. If $f(z) \in \mathcal{K}(\theta, \alpha)$, then

$$
\sum_{n=2}^{\infty} n(n-\alpha)\left|a_{n}\right| \leq 1-\alpha
$$

Proof. Let $f(z) \in \mathcal{K}(\theta, \alpha)$. Then, we have

$$
\begin{aligned}
\operatorname{Re}\left\{1+\frac{z f^{\prime \prime}(z)}{f^{\prime}(z)}\right\} & =\operatorname{Re}\left\{\frac{1+\sum_{n=2}^{\infty} n^{2} a_{n} z^{n-1}}{1+\sum_{n=2}^{\infty} n a_{n} z^{n-1}}\right\} \\
& =\operatorname{Re}\left\{\frac{1-\sum_{n=2}^{\infty} n^{2}\left|a_{n}\right| e^{i(n-1) \theta} z^{n-1}}{1-\sum_{n=2}^{\infty} n\left|a_{n}\right| e^{i(n-1) \theta} z^{n-1}}\right\}>\alpha
\end{aligned}
$$

for all $z \in \mathcal{U}$. Let us consider a point $z \in \mathcal{U}$. such that $z=|z| e^{-i \theta}$.

Then we have

$$
\frac{1-\sum_{n=2}^{\infty} n^{2}\left|a_{n}\right||z|^{n-1}}{1-\sum_{n=2}^{\infty} n\left|a_{n}\right||z|^{n-1}}>\alpha
$$

Letting $|z| \rightarrow 1^{-}$, we obtain the inequality (4.1).

Corollary 4.2. If $f(z) \in \mathcal{K}(\theta, \alpha)$, then

$$
\left|a_{n}\right| \leq \frac{1-\alpha}{n(n-\alpha)} \quad(n=2,3, \ldots) .
$$

Remark 4.3. If $f(z) \in \mathcal{K}(\theta, \alpha)$, then

$$
\sum_{n=2}^{\infty} n(n-1)\left|a_{n}\right| \leq \sum_{n=2}^{\infty} n(n-\alpha)\left|a_{n}\right| \leq 1-\alpha .
$$

Applying Theorem 2.1, Lemma 4.1 and using the same technique as in the proof of Theorem 3.4, we derive 
Theorem 4.4. Let $f(z) \in \mathcal{K}(\theta, \alpha)$, and $\delta \in \mathbb{C}(0<|\delta|<1)$. Then the function $\frac{1}{\delta} f(\delta z) \in \mathcal{T}\left(\beta_{1}, \beta_{2}, \beta_{3} ; \lambda\right)$ for $\left(0<|\delta| \leq\left|\delta_{0}(\lambda)\right|\right.$, where $\left|\delta_{0}(\lambda)\right|$ is the smallest positive root of the equation

$$
\begin{aligned}
& \left|\beta_{1}\right| \frac{|\delta| \sqrt{2(1-\alpha)}}{\left(1-|\delta|^{2}\right)^{3 / 2}}+\left|\beta_{2}\right| \frac{|\delta|^{2} \sqrt{\left(6+18|\delta|^{2}\right)\left(1-\alpha-2\left|a_{2}\right|^{2}\right)}}{\left(1-|\delta|^{2}\right)^{5 / 2}} \\
& +\left|\beta_{3}\right| \frac{4 \sqrt{3}|\delta|^{3} \sqrt{\left(1+8|\delta|^{2}+6|\delta|^{4}\right)\left(1-\alpha-2\left|\alpha_{2}\right|^{2}-6\left|a_{3}\right|^{2}\right)}}{\left(1-|\delta|^{2}\right)^{7 / 2}}=\lambda
\end{aligned}
$$

in $0<|\delta|<1$.

Competing interests

The author declares that they have no competing interests.

Received: 13 July 2011 Accepted: 9 February 2012 Published: 9 February 2012

\section{References}

1. Duren, PL: Univalent Functions. Springer-Verlag, Berlin (1983)

2. Goodman, AW: Univalent Functions. Mariner, Tampa1-2 (1983)

3. Uyanik, N, Owa, S: New extensions for classes of analytic functions associated with close-to-convex and starlike of order a. Math Comput Model. 54, 359-366 (2011). doi:10.1016/j.mcm.2011.02.020

doi:10.1186/1029-242X-2012-24

Cite this article as: Frasin: New subclasses of analytic functions. Journal of Inequalities and Applications 2012 2012:24.

\section{Submit your manuscript to a SpringerOpen ${ }^{\odot}$} journal and benefit from:

Convenient online submission

Rigorous peer review

- Immediate publication on acceptance

- Open access: articles freely available online

- High visibility within the field

- Retaining the copyright to your article

Submit your next manuscript at $\boldsymbol{\wedge}$ springeropen.com 